\section{$\underset{\text { hommes migrations }}{\text { homments }}$}

\section{Hommes \& migrations}

Revue française de référence sur les dynamiques

migratoires

$1296 \mid 2012$

Le Mexique dans les migrations internationales

\title{
Mexicaines aux états-Unis, Espagnoles en France (1950-1970)
}

\section{Blanca Cecilia Ceceña Camacho}

\section{(2) OpenEdition \\ Journals}

\section{Édition électronique}

URL : http://journals.openedition.org/hommesmigrations/1519

DOI : 10.4000/hommesmigrations.1519

ISSN : 2262-3353

Éditeur

Musée national de l'histoire de l'immigration

\section{Édition imprimée}

Date de publication : 1 mars 2012

Pagination : 114-117

ISSN : 1142-852X

Référence électronique

Blanca Cecilia Ceceña Camacho, « Mexicaines aux états-Unis, Espagnoles en France (1950-1970)», Hommes \& migrations [En ligne], 1296 | 2012, mis en ligne le 29 mai 2013, consulté le 22 septembre 2020. URL : http://journals.openedition.org/hommesmigrations/1519; DOI : https://doi.org/10.4000/ hommesmigrations.1519

Ce document a été généré automatiquement le 22 septembre 2020

Tous droits réservés 


\title{
Mexicaines aux états-Unis, Espagnoles en France (1950-1970)
}

\author{
Blanca Cecilia Ceceña Camacho
}

1 Les migrations de l'Espagne vers la France et du Mexique vers les États-Unis ont donné lieu à de nombreux travaux ${ }^{1}$. Néanmoins les recherches se sont moins intéressées au cas des femmes ${ }^{2}$. De plus, l'étude comparée des migrations reste un champ de recherche marginal ${ }^{3}$. C'est pourquoi le travail proposé ici tente d'effectuer une étude comparée de l'histoire des femmes entre les années cinquante et soixante-dix. Ces années ont connu une vague migratoire considérable d'Espagnols vers la France et de Mexicains vers les États-Unis ${ }^{4}$. La méthode employée ici consiste à travailler à partir d'entretiens réalisés auprès de femmes espagnoles et mexicaines parties vers la France et vers les États-Unis entre les années cinquante et soixante-dix. Ce travail a été mené en parallèle avec une analyse sur les recherches concernant les populations migrantes en question, sans oublier ce qui concerne la structure de la population active en France et aux États-Unis ${ }^{5}$.

\section{Le développement structurel et l'appel à l'immigration}

Durant les années cinquante à soixante, les économies américaine et française sont engagées dans un développement structurel qui fait appel à l'immigration massive, tel est le cas en France de l'agglomération parisienne et aux États-Unis de celle de Los Angeles.

\section{La France : l'exemple de Paris}

Entre 1950 et 1969, le niveau de développement économique et le poids sur la scène internationale de la France et de l'Espagne sont très différents ${ }^{6}$. La France est engagée dans un processus d'expansion économique qui puise ses racines dans la période de l'après-guerre. Dans les années cinquante, malgré le déficit commercial, l'inflation et les troubles sociaux, le pays réalise des progrès spectaculaires. Au cours de la décennie 
suivante, la croissance s'accélère. Dans les années soixante, le pays bénéficie donc d'une solide position structurelle. L'Espagne a tiré les conséquences d'une longue période d'isolement et d'autarcie. Sa situation commence à changer avec le plan de stabilisation de 1959 et l'entrée du pays dans certains des plus importants organismes économiques internationaux (le FMI, l'OECE, la Banque mondiale). Cependant, l'Espagne souffre encore d'un manque de libéralisation politique ${ }^{7}$. De 1950 à 1970, la vie parisienne évolue. Si la question du logement reste préoccupante dans la décennie soixante, la prospérité économique permet à l'ensemble des Parisiens d'accroître leur pouvoir d'achat. Les catégories sociales se modifient. Les ouvriers, encore nombreux dans les années cinquante, sont progressivement remplacés par les employés du fait de la tertiarisation de l'économie dans les années soixante-dix. Le travail à la chaîne, le recours aux équipes alternées, le travail de nuit, l'usage des heures supplémentaires et la main-d'œuvre abondante et bon marché, grâce à l'apport de travailleurs étrangers, ont été les moteurs de cette prospérité économique. Les Espagnols remplacent la vague italienne qui s'estompe ${ }^{8}$. Au début des années soixante-dix, les Portugais sont privilégiés par l'État français qui prévoit de remplacer la vague espagnole amorçant son déclin. Le niveau de vie des Parisiens augmente. S'ils connaissent la société de consommation, les exclus de la richesse sont nombreux. Beaucoup sont des immigrés, nouvellement arrivés avec des salaires très bas et des conditions de travail difficiles voire dangereuses. Il subsiste un chômage constant, bien que faible, touchant en priorité ces exclus. En région parisienne, en 1970, cent treize bidonvilles regroupent 46000 personnes'. L'intégration des étrangers se fait difficilement quelles que soient les vagues d'immigration. Cette immigration déqualifie l'étranger avant de lui donner sa chance. L'exemple des Italiens dans les années vingt montre comment Paris gâche cette inépuisable ressource de main-d'œuvre. Ce gaspillage existe pour les Espagnols qui ont subi de plein fouet une pareille déqualification à leur arrivée ${ }^{10}$. Les récurrences sont à étudier. Les Espagnols à Paris ont constitué une vague d'immigration qui s'insère dans une histoire parisienne se nourrissant de ces immigrations successives ${ }^{11}$. L'étude du vécu des Espagnols dans la banlieue ouvrière nord de la Plaine-Saint-Denis a montré une adaptation réelle, mais aussi les nombreuses difficultés qu'ils ont eu à affronter ${ }^{12}$.

\section{Les États-Unis : l'exemple de Los Angeles}

4 À la crise de 1929 a succédé un extraordinaire développement industriel et démographique, dont témoigne Los Angeles. Dès cette époque, elle s'est imposée comme la cinquième métropole des États-Unis. En même temps, pendant les années soixante, le barrio de l'est de Los Angeles est devenu l'aire la plus habitée par les Mexicains aux États-Unis ${ }^{13},{ }^{14}$.

5 Depuis que la ville appartient aux États-Unis ${ }^{15}$, une politique de ségrégation sociale s'est installée au détriment des Mexicains. Les Anglo-Américains restreignent le vote mexicain, mettent en place l'interdiction des pratiques culturelles mexicaines. Malgré la crainte de la "dégénérescence raciale" des Anglo-Américains, les Mexicains fournissent la main-d'œuvre la plus adaptée à l'économie régionale. Beaucoup des migrants spanish speaking ont trouvé du travail dans des postes semi-qualifiés ou déqualifiés acceptant ainsi bas salaires, surmenage et mauvaises conditions d'emploi ${ }^{16}$. 
Grâce à la maturité des réseaux sociaux transnationaux informels et, en particulier, au développement de réseaux de femmes, beaucoup d'entre elles ont bénéficié d'offres d'emplois américains dans les usines, les hôtels et les résidences privées ${ }^{17}$.

\section{Femmes migrantes et l'offre d'emploi}

7 D'après notre enquête, la trajectoire de travail et les expériences liées à celle-ci sont similaires chez les femmes espagnoles et mexicaines migrantes pendant les années cinquante et soixante-dix.

Pendant ce temps-là, le Mexique et l'Espagne connaissent une crise structurelle qui a touché particulièrement la campagne en raison d'une urbanisation accélérée et non maitrisée. Cherchant une meilleure qualité de vie pour elles et pour les leurs, les femmes quittent leur foyer. Le premier travail qu'elles trouvent est souvent un poste de domestique.

9 Aucune des femmes interviewées qui sont arrivées dans le pays d'accueil de façon légale ou illégale ne voulait être "bonne" ou "live-in house keeper". Personne ne veut voir ses filles exerçant ce genre de travail et toutes veulent quitter la profession. Elles sont fières de la qualité de leur travail et de pouvoir subvenir aux besoins de leur famille. Pourtant, elles ne sont pas fières d'être travailleuses domestiques. Cette autodistanciation des Mexicaines vis-à-vis de leur statut professionnel rend plus difficile le fait de voir ce travail salarié comme un véritable emploi.

10 Au travail, à la maison de l'employeur, le rapport de pouvoir exprimé par les employeurs réintroduit l'inégalité ethnique et de classe chez les femmes. La relation entre l'employée et l'employeur, telle qu'elle prévaut aujourd'hui, fait plus qu'exacerber les inégalités en niant aux travailleurs domestiques les plus modestes toutes formes de reconnaissance sociale.

\section{Conclusion}

11 À la différence du développement du marché de l'emploi domestique en France concernant les employées espagnoles, aux États-Unis le travail domestique est réalisé principalement par des migrants illégaux. La moitié des quatre millions d'adultes migrantes engagées travaillent au noir et viennent principalement du Mexique. Le problème subsiste encore aujourd'hui. Aux États-Unis, l'analyse du recensement montre que, de 1980 à 1990, la population travaillant comme domestique a doublé18. Pendant les années quatre-vingt-dix, les droits et privilèges sont inférieurs pour les résidents permanents légaux et les citoyens naturalisés dans la mesure où les campagnes contre l'immigration clandestine se trouvent à l'origine d'attaques xénophobes à l'égard de tous les migrants, y compris ceux qui bénéficient d'une autorisation légale. Comme exemples des initiatives américaines pour la ségrégation des migrants, il y a la Proposition 187 et l'Opération "Guardian" en Californie en 1994 et, plus récemment, la loi SB 1070 en Arizona (2010). Le statut de migrant est clairement devenu un axe majeur d'inégalité, en rapport avec la nationalité, la classe et le genre, ce qui facilite l'exploitation des travailleurs migrants.

12 Les chances de reconnaissance au travail auraient pu être améliorées chezles Mexicaines s'il y avait eu une forte réglementation du marché de l'emploi, et si les 
travailleuses domestiques avaient bénéficié d'une organisation collective, comme celle mise en place par les Espagnols (hommes et femmes). Pour que cela soit efficace, ces efforts doivent se produire en parallèle avec une nouvelle reconnaissance des rapports de travail dans les relations domestiques salariées.

\section{NOTES}

1. Émile Temine, "Le rôle de la communauté espagnole en France. Une problématique d'ensemble", in Relations internationales, $\mathrm{n}^{\circ}$ 50, 1987, pp.33-52; Ana Fernandez Asperilla, "Estrategias migratorias a partir del proceso de la emigracion española en Europa (1959-2000)", in Migraciones y Exilios, $\mathrm{n}^{\circ} 1$, 2000, pp.67-94; Rodolfo Tuiran, Migracion México-Estados Unidos: Continuidad y Cambio, México, Conapo, 1970 ; Emory Bogardus, The mexican in the United States, Los Angeles, University of Southern California Press, 1970.

2. Laura Oso Casas, Españolas en Paris. Estrategias de ahorro y consumo en las migraciones internacionales, Barcelona, Bellaterra, 2004 ; Pierrette Hondagneu-Sotelo, Doméstica: Immigrant Workers Cleaning and Caring in the Shadows of Affluence, Berkeley and Los Angeles, University of California Press, 2001.

3. Deborah Cohen et O'Connor Maura (dir.), New York, Routledge, 2004, pp. 41-56 ; Nancy Green, “Forms of Comparison", in Deborah Cohen and O'Connor Maura (dir.), Comparison and History: European Cross-National Perspective, New York, Routledge, 2004, pp. 41-56 ; Jean-Marie Hannick, Simples réflexions sur l'histoire comparée, Louvain-la-neuve, Folia Electronica Clasica, 2001.

4. Pour mener à bien la recherche, deux types de sources sont disponibles. Il s'agit d'abord de sources primaires, essentiellement des archives et des statistiques officielles: Centres de référence pour la documentation. France: Institut national d'études démographiques (Ined), Archives nationales à Fontainebleau; Mexique : Instituto Nacional de Migracion (Inami); ÉtatsUnis: UCLA Chicano Studies Research Center; et les archives de l'État de la Californie : State Archives in Sacramento, Californie. Auxquelles s'ajoutent des histoires de vie et des enquêtes : vingt-cinq entretiens avec des femmes espagnoles en France et vingt-cinq avec des mexicaines aux États-Unis. Ces interviews ont été conduites par Blanca Ceceña pendant les années 2008-2010. 5. Statistiques de population faites par l'Ined en France et par le U.S. Census Bureau aux ÉtatsUnis.

6. Esther Sanchez, Rumbo al Sur: Francia y España del desarrollo, 1958-1969, Madrid, Biblioteca de Historia, Consejo Superior de Investigaciones Cientificas, Ministerio de Educacion y Ciencia, 2006, p. 396.

7. Ibid.

8. Alexis Spire, Étrangers à la carte. L'administration de l'immigration en France, Paris, Grasset, 2005, p. 111 ; Yvan Gastaut, L'Immigration et l'opinion en France sous la Ve République, Paris, Seuil, 2000, p. 78 .

9. Pascale Gauthier, Les Espagnols à Paris et le Service social d'aide aux émigrants, de 1945 aux années 1980. Le parcours des Esparisiens dans la capitale, Paris, École des hautes études en sciences sociales, 2008, pp. 21-22.

10. Ibid. ; Alexis Spire, Étrangers à la carte. L'administration de l'immigration en France, op. cit. ; Yvan Gastaut, L'Immigration et l'opinion en France sous la Ve République, op. cit. 
11. Pascale Gauthier, Les Espagnols à Paris et le Service Social d'Aide aux Emigrants, de 1945 aux années 1980. Le parcours des Esparisiens dans la capitale, op. cit.

12. Natacha Lillo, "Espagnols en banlieue rouge, histoire comparée de trois vagues migratoires à Saint Denis et dans sa région au XXe siècle", thèse de doctorat, sous la direction de Pierre Milza, Paris, IEP, 2001.

13. "Cette aire, avec plus de 90000 résidents mexicains, dépasse celle d'Albany (New York), Sacramento (Californie) et Austin (Texas), villes capitales de trois des régions les plus grandes des Etats-Unis". Ricardo Romo, History of a Barrio, East Los Angeles, Austin, Austin University Press, p. 5.

14. Ibid.

15. En 1810, avec l'indépendance du Mexique vis-à-vis de l'Espagne, la région de la Californie appartient au Mexique. Cette période est caractérisée par les divergences politiques et culturelles dans le territoire. En outre, les États-Unis avaient une volonté d'expansion territoriale et économique (notamment de l'industrie d'extraction de minerais) dans ces terres. Cette période a culminé avec la guerre entre le Mexique et les États-Unis. Celle-ci a pris fin avec la signature d'un traité, nommé Guadalupe Hidalgo, par lequel le Mexique cède la Californie à son voisin du nord.

16. Ricardo Romo, History of a Barrio, East Los Angeles, op. cit.

17. Douglas Massey, Rafael Alarcon, Jorge Durand et Humberto Gonzalez, Return to Aztlan: The Social Process of International Migration from Western Mexico, Berkeley, University of California Press, 1987 ; Pierrette Hondagneu-Sotelo, "Regulating the Unregulated: Domestic Workers' Social Networks", in Social Problems, n 41, 1994, pp. 201-215; Lourdes Beneria et Roldan Roldan, The Crossroads of Class and Gender : Industriel Homework, Subcontracting, and Household Dynamics, Chicago, University of Chicago Press, 1987; Orlandina De Oliveira, "Empleo femenino en México en tiempos de recesion economica: Tendencias Recientes", in Mujer y crisis: Respuestas ante la recesion, Caracas, Neuma Aguilar-Editorial Nueva Sociedad, 1990, pp. 31-54.

18. Pierrette Hondagneu-Sotelo, "Regulating the Unregulated: Domestic Workers' Social Networks", op. cit.

\section{AUTEUR}

\section{BLANCA CECILIA CECEÑA CAMACHO}

Doctorante en histoire contemporaine, Paris-Sorbonne, Paris IV, École doctorale II, Histoire moderne et contemporaine UMR 8596 - Centre Roland-Mousnier. 